\title{
Effects of the Tangningtongluo formula as an alternative strategy for diabetics via upregulation of insulin receptor substrate-1
}

\author{
LONG CHENG ${ }^{1,2^{*}}$, JUNMEI SONG ${ }^{3}$, GENG LI $^{4}$, YUE LIU $^{5}$, YUMING WANG $^{6}$, XIANGBAO MENG $^{1 *}$, \\ GUIBO SUN ${ }^{1}$ and XIAOBO SUN ${ }^{1}$
}

\begin{abstract}
${ }^{1}$ Pharmacology and Toxicology Center, Institute of Medicinal Plant Development, Chinese Academy of Medical Sciences and Peking Union Medical College, Beijing 100193; ${ }^{2}$ Key Laboratory of Pharmacology, Zunyi Medical University, Zunyi, Guizhou 563003; ${ }^{3}$ Department of Clinical Pharmacy, Baoji People's Hospital, Baoji, Shaanxi 721000;

${ }^{4}$ Integrative Medicine Center for Cardiovascular Disease, China-Japan Friendship Hospital, Beijing 100029;

${ }^{5}$ Cardiovascular Disease Centre, Xiyuan Hospital, China Academy of Traditional Chinese Medicine, Beijing 100091;

${ }^{6}$ Department of Oncology, The Affiliated Hospital of Chifeng University, Chifeng, Inner Mongolia 024005, P.R. China
\end{abstract}

Received May 31, 2016; Accepted April 6, 2017

DOI: $10.3892 / \mathrm{mmr} .2017 .6679$

\begin{abstract}
Tangningtongluo (TNTL), a traditional Chinese medicine, has been widely used in clinics for decades in southwest China. Its pharmacological properties and underlying molecular mechanisms remain unclear. The main goal of ethnopharmacology is to identify novel bioactive compounds derived from plants for use in indigenous medical practice. This knowledge can be used to develop novel pharmaceuticals. In the present study, hyperglycemic C57BL/KsJ-db/db $(\mathrm{db} / \mathrm{db})$ mice were used to test the effect of TNTL on microvasculature of the retina and hypoglycemia. Metformin (Met) was selected as a positive control. 26-week-old mice were randomly assigned to receive either the anti-diabetic agent Met $[140 \mathrm{mg} / \mathrm{kg}$ body weight (BW)], $1.8,0.9$ or $0.45 \mathrm{~g} / \mathrm{kg}$ BW TNTL, or a placebo. The fasting blood glucose, serum insulin and glycated hemoglobin levels were measured. Histopathologic examination of the pancreas was performed
\end{abstract}

Correspondence to: $\mathrm{Dr}$ Xiaobo Sun or Dr Guibo Sun, Pharmacology and Toxicology Center, Institute of Medicinal Plant Development, Chinese Academy of Medical Sciences and Peking Union Medical College, 151 Malianwa North Road, Beijing 100193, P.R. China

E-mail: sun-xiaobo@163.com

E-mail: sunguibo@163.com

*Contributed equally

Abbreviations: TNTL, Tangningtongluo; TCM, traditional Chinese medicine; db/db, C57BL/KsJ-db/db, T2DM, type 2 diabetes mellitus; IDF, International Diabetes Federation; HbAlc, glycated hemoglobin A1c; Met, metformin; HOMA-IR, homeostatic model assessment of insulin resistance

Key words: hypoglycemic effect, diabetes mellitus, insulin receptor substrate-1, traditional Chinese medicine, Tangningtongluo to confirm the hypoglycemic effect. Fluorescein angiography was applied to detect diabetes-induced retinal angioma in the $\mathrm{db} / \mathrm{db}$ mice. TNTL intake significantly decreased the fasting blood glucose level in a dose-dependent manner. Additionally, TNTL intervention resulted in a significant decrease in the insulin resistance index. Notably, TNTL treatment markedly reduced the speed of retinal degeneration and mildly reversed microvascular caliber degeneration. Western blot analysis indicated that upregulation of phosphorylated insulin receptor substrate-1 (p-IRS-1) by the administration of TNTL may be strongly involved in the improvement of insulin resistance. In conclusion, TNTL exerted a strong hypoglycemic effect and reversed retinal degeneration via upregulation of ISR-1. The present findings provide important scientific evidence supporting TNTL as an effective alternative approach for the management of Type 2 diabetes mellitus.

\section{Introduction}

The prevalence of diabetes, especially type 2 diabetes mellitus (T2DM), is increasing in China and worldwide (1-5). The International Diabetes Federation (IDF) predicts that the global prevalence of diabetes will grow from 382 million in 2013 to 592 million by 2035 (5). China is now home to a quarter of the world's diabetes sufferers, which amounts to $>100$ million people-nearly $12 \%$ of the population $(3,6,7)$. More than 600 million Chinese people suffer from prediabetes (7). These are epidemic proportions, and China may face difficulties controlling its crisis compared with other more developed nations $(8,9)$. Therefore, urgent action is required $(2,4,10)$.

Traditional Chinese medicine (TCM) has served an important role in the healthcare system in the past $(11,12)$. At present, TCM is still well integrated in the Chinese health-care system as one of the two mainstream medical practices due to economic, cultural and historical evolutionary determinants (13-17). Despite the fact that TCM may possess synergistic anti-diabetic effects, a systematic evidence-based strategy to improve T2DM treatment and/or reduce the risk 
of complications is still not available. Nevertheless, several preclinical and clinical trials were performed to collect evidence on the benefits of TCM (17-21). One TCM, Liu Wei Di Huang Wan, or Six Flavor Rehmanni, has been demonstrated to possess a particular effect on diabetes or diabetic complications (22). In the clinical setting, TCMs are often used combined with Western medicine, and a clinical study demonstrated that the combined effect is an improvement on the use of Western medicine alone (23). There are several advantages in treating DM with TCM, including lower rates of toxicity and/or side effects, holistic regulation of metabolic problems, reversal of risk factors leading to T2DM and delaying diabetic complications.

Tangningtongluo (TNTL), a TCM, has been widely used for decades in southwest China. The traditional medical formula is composed of Herba Plantagins, kewoluoqu, Flos Lonicerae and Herba Agrimoniae. In 2014, the preparation of TNTL was authorized by the local drug administration department of Guizhou Provincial Food and Drug Administration (Guiyang, China; authorization no. QYZZ-2014033). The aim of the present study was to analyze the effectiveness of the TNTL treatment strategy and to investigate the associated hypoglycemic mechanism.

\section{Materials and methods}

Preparation of the plant material. Plant material was collected from the Chinese Herbal Medicine Planting Base of Guizhou Bailing Pharmaceutical Co., Ltd. (Guizhou, China). This research was permitted by the director of the planting base. Plant material was botanically authenticated and a voucher specimen (no. BL-130012 130015) was deposited in the Miao Medicine Herbarium of Guizhou Bailing Pharmaceutical Co., Ltd. The company also provided an aqueous extract of the TNTL formula. To obtain the aqueous extract, the herb formula [Herba Plantagins (Cheqiancao), $1.16 \mathrm{~kg}$; kewoluoqu, $1.6 \mathrm{~kg}$; Flos Lonicerae (Shanyinghua), $1.11 \mathrm{~kg}$; and Herba Agrimoniae (Xianhecao), $1.11 \mathrm{~kg}$ ] was cleaned and extracted with hot water, yielding a powdered extract $(1.0 \mathrm{~kg}$ TNTL extract, $20.1 \%$ extract percentage), which was used for bioassay. To reduce the variability of TNTL among different batches, the species, origin, harvest time, medicinal parts and concocted methods for each component were strictly standardized according to good manufacturing practice. Furthermore, for quality control, a fingerprint of TNTL was established using the high-performance liquid chromatography technique in our previous study (24). In 2014, the preparation of TNTL was authorized by the local drug administration department (authorization no. QYZZ-2014033).

Chemical reagents. A One-Touch Ultra Blood Glucose Meter and strips (cat no. 3462320) were obtained from Johnson \& Johnson Medical Equipment Co., Ltd. (Shanghai, China). A carboxyl methyl cellulose $(0.5 \%)$ solution served as the vehicle. Metformin (Met; Squib Pharmaceutical Co., Ltd., Shanghai, China), suspended in $0.5 \%$ carboxyl methyl cellulose solution was used as the reference drug for hypoglycemic activity measurement. Insulin receptor substrate-1 (ISR-1; ab52167), phosphorylated (p)-ISR-1 (phospho S522; ab65745) and $\beta$-actin (ab8227) primary antibodies, goat anti-Rabbit IgG
H\&L (HRP) (ab6721) and goat anti-mouse IgG H\&L (HRP; ab6789) second antibodies were purchased from Abcam (Cambridge, MA, USA).

Experimental animals. To confirm the hypoglycemic activity and the effect on pre-proliferative diabetic retinopathy, 6-week-old female C57BL/KsJ-db/db mice (specific pathogen free; $n=50 ; 45-50 \mathrm{~g}$ ) were purchased from SLAC Laboratories Animal Co., Ltd. (Shanghai, China) for a T2DM model $(25,26)$. C57BL female mouse (specific pathogen free $n=10,5-6$ weeks, 18-22 g) were purchased from SLAC Laboratories Animal Co., Ltd. (Shanghai, China) as a normal control group. The animals were housed under controlled conditions (temperature, $23 \pm 2^{\circ} \mathrm{C}$; relative humidity, $50 \pm 10 \%$; 12 -h light/dark cycle) and allowed free access to a standard diet. All mice were acclimated for 20 weeks for the emergence of pre-proliferative diabetic retinopathy prior to the initiation of the drug intervention and were maintained according to Beijing Laboratory Animal Management Regulations. The experiment protocol was reviewed and approved by the Animal Management Committee of the Animal Resource Center, Institute of Medicinal Plant Development, Chinese Academy of Medical Sciences (Beijing, China).

Experimental protocol. Fluorescein angiography (FA) was applied to detect early diabetes-induced alterations in the microvasculature of the retina with an Animal Fluorescein Imaging system (ISSLO/SOS2000; Optoprobe Research Ltd., Burnaby, BC, Canada), including a 4D-isOCT Retinal Microscope Imaging system (Optoprobe ${ }^{\circledR}$; Optoprobe Research Ltd.). After detection of the microvascular hemangioma of diabetic retinopathy, 26-week-old $\mathrm{db} / \mathrm{db}$ mice were randomly divided into 5 groups, each containing 10 mice. The C57BL mouse group (group 1, normal control group) and $\mathrm{db} / \mathrm{db}$ diabetic controls (group 2, model control group, placebo) received an equal volume of vehicle. Positive controls (group 3, model + Met group) received the anti-diabetic agent, Met $[140 \mathrm{mg} / \mathrm{kg}$ body weight (BW), an equivalent dose]. TNTL-treated mice received $1.8 \mathrm{~g}, 0.9 \mathrm{~g}$ or $0.45 \mathrm{~g} / \mathrm{kg} \mathrm{BW}$ (groups 4,5 and 6 , respectively; model + TNTL groups). In clinic practice, the recommend dose was 2.7 3.6 g per subject daily, which is $\sim 0.045 \mathrm{~g} / \mathrm{kg} \mathrm{BW}$ for an adult (average BW $=60 \mathrm{~kg}$ ). The equivalent dose in mice was $0.45 \mathrm{~g} / \mathrm{kg}$. In the present study, doses that were 2 -fold $(0.9 \mathrm{~g} / \mathrm{kg}), 4$-fold $(1.8 \mathrm{~g} / \mathrm{kg})$ and an equivalent dose $(0.45 \mathrm{~g} / \mathrm{kg})$ were administered orally per day continuously for 18 weeks in mice to evaluate the activity. All mice received the assigned intervention daily and $\mathrm{BW}$, food and water intake were measured weekly.

Blood glucose determination. To determine fasting blood glucose levels, blood samples were drawn from the tail vein of all mice every week. After placing fresh blood $(\sim 50 \mu \mathrm{l})$ on duplicate test strips, a validated One-Touch Basic Glucose Monitoring system was used to determine the glucose content. After 18 weeks of intervention, all animals were fasted for $5 \mathrm{~h}$ and anesthetized with diethyl ether (inhalation dosage: 2.0\% for induction, $0.2-0.3 \%$ for maintenance; Sigma-Aldrich; Merck KGaA, Darmstadt, Germany). After injecting fluorescein $(20 \mu \mathrm{l})$ into the tail vein of the mice, FA was performed to detect early diabetes-induced alterations in microvasculature 
with an Animal Fluorescein Imaging system and a 4D-isOCT Retinal Microscope Imaging system. Subsequently, the blood samples were collected for biochemical analysis.

Biochemical analysis. Blood samples were centrifuged at $3,000 \times \mathrm{g}$ for $15 \mathrm{~min}$ at $4^{\circ} \mathrm{C}$ and then removed and stored the plasma $\left(-60^{\circ} \mathrm{C}\right)$ for further analysis. The plasma insulin and C-peptide levels were detected immediately using a radioimmunoassay. Commercial kits and a standard assay method were used to estimate the plasma glycated hemoglobin Alc (HbAlc; EXP210563) level and the plasma lipid profile of triacylglycerol (TG; EXP210617), total cholesterol (TC; EXP210602), high-density lipoprotein (HDL) and low-density lipoprotein (LDL; EXP210603) (all from Beijing Expandbiotech Co., Ltd., Beijing, China).

The homeostatic model assessment of insulin resistance (HOMA-IR) index was applied to estimate the alteration in insulin resistance in TNTL-treated diabetic mice. The HOMA-IR calculation was performed using the following formula: HOMA-IR = fasting blood glucose $(\mathrm{mmol} / \mathrm{l}) \mathrm{x}$ insulin $(\mu \mathrm{U} / \mathrm{ml}) / 22.5(27)$.

Protein extraction and western blot analysis. To determine the protein expression level associated with insulin resistance, the liver and gastrocnemius muscle tissue were homogenized $(1: 10 \mathrm{w} / \mathrm{v})$ on ice with radioimmunoprecipitation assay buffer [50 mM Tris (pH 7.6), $150 \mathrm{mM} \mathrm{NaCl}, 1 \%$ Triton $\mathrm{X}-100,1 \%$ sodium deoxycholate, $0.1 \%$ sodium dodecyl sulfate (SDS) and 2 mM EDTA; sterile solution] containing protease and phosphatase inhibitors (26). Following this, the supernatants were collected after centrifugation at $12,000 \mathrm{x}$ g for $25 \mathrm{~min}$ at $4^{\circ} \mathrm{C}$. A bicinchoninic acid assay was used to determine the protein concentration and the protein was stored at $-80^{\circ} \mathrm{C}$ for further western blot analysis. The protein fractions were separated by electrophoresis on $10 \%$ SDS-PAGE gels and then transferred onto nitrocellulose membranes in Tris-glycine buffer at $110 \mathrm{~V}$ for $1 \mathrm{~h}$. The membranes were blocked with $5 \%(\mathrm{w} / \mathrm{v})$ skimmed milk powder in Tris-buffered saline containing $0.1 \%(\mathrm{v} / \mathrm{v})$ Tween-20 (TBST) and then incubated overnight with ISR-1, p-ISR-1 and $\beta$-actin primary antibodies $(1: 1,000)$ at $4^{\circ} \mathrm{C}$. Following this, the membranes were washed twice with TBST and incubated with secondary antibodies (goat anti-mouse IgG H\&L; ab6789; 1:1,000; Abcam) for $2 \mathrm{~h}$ at room temperature. The results were visualized by an Enhanced Chemiluminescence kit (Thermo Scientific, Inc., Waltham, MA, USA). The relative band intensity was determined using a computerized densitometric analysis (Bio Image Intelligent Quantifier, version, IQ-11; Bio Image Systems, Inc., Jackson, MI, USA).

Histopathological examination. Following the oral glucose tolerance test, all mice were anesthetized with diethyl ether (10\% inhalation dosage) and sacrificed by cervical decapitation. The liver, kidney, pancreas and gastrocnemius muscle tissue were quickly removed for examination by light microscopy. The tissue sections were preserved in $10 \%$ neutral phosphate-buffered formalin and processed by routine paraffin sectioning $(2 \mu \mathrm{m})$ and staining with $\mathrm{H} \& \mathrm{E}$. Staining was performed according to the manufacturer's protocol. Pathological alterations were observed under an optical microscope using a Leica Application Suite (Leica Microsystems, Inc., Buffalo Grove, IL, USA).

Statistical analysis. Statistical analysis was performed with SPSS software version 11.5 (SPSS Inc., Chicago, IL, USA). All data are expressed as the mean \pm standard deviation. Comparisons between groups were analyzed by one-way analysis of variance and group comparisons (the post hoc multiple comparison) were analyzed using the Student-Newman-Keuls test, if equal variances assumed or Thamhan's T2, if equal variances not assumed. The paired t-test was used for continuous variables in the retrospective analysis. $\mathrm{P}<0.05$ was considered to indicate a statistically significant difference.

\section{Results}

Effect of TNTL on the metabolic abnormalities in $\mathrm{db} / \mathrm{db}$ mice. As presented in Fig. 1, the TNTL intervention resulted in a significant time-dependent decrease in fasting blood glucose in $\mathrm{db} / \mathrm{db}$ mice from week 1 to 18 . Compared with the placebo, TNTL (1.8, 0.9 and $0.45 \mathrm{~g} / \mathrm{kg} \mathrm{BW})$ or Met $(140 \mathrm{mg} / \mathrm{kg} \mathrm{BW})$ treatment did not significantly affect the BW, food and water intake or feed efficiency ratio of the $\mathrm{db} / \mathrm{db}$ mice (data not shown). As presented in Fig. 2, the TNTL intervention resulted in a significant dose-dependent decrease in HbA1c level $(5.50 \pm 1.06,5.02 \pm 0.56$ and $4.80 \pm 0.63$ vs. $7.12 \pm 0.78$ for $0.45,0.9$ and $1.8 \mathrm{~g} / \mathrm{kg} \mathrm{BW}$ doses of TNTL vs. placebo, respectively). As expected, Met significantly lowered the levels of blood glucose (Fig. 1) and HbAlc (5.78 \pm 0.72 vs. $7.12 \pm 0.78$; Fig. 2) in $\mathrm{db} / \mathrm{db}$ mice. Therefore, TNTL exhibits a time-dependent hypoglycemic effect with sustained medication.

Serum lipid levels of TG, TC and HDL were increased significantly in the model group compared with normal mice, indicating dyslipidemia in $\mathrm{db} / \mathrm{db}$ diabetic mice. Compared with the diabetic control group, the TNTL treatment markedly reduced plasma concentrations of TG, TC and LDL in a dose-dependent manner (Table I).

The plasma insulin and C-peptide levels, as assessed by HOMA-IR index, increased significantly in $\mathrm{db} / \mathrm{db}$ mice compared with the normal control group. Treatment with Met resulted in a significant decrease in the HOMA-IR index compared with the $\mathrm{db} / \mathrm{db}$ model $(12.86 \pm 8.91$ vs. $30.61 \pm 5.43$. TNTL treatment $(1.8,0.9$ and $0.45 \mathrm{~g} / \mathrm{kg} \mathrm{BW})$ resulted in the same effect compared with the $\mathrm{db} / \mathrm{db}$ model group $(10.55 \pm 1.55,11.85 \pm 2.56$ and $11.23 \pm 4.693$ vs. $30.61 \pm 5.43$, respectively; Fig. 3).

Effect of TNTL on retinal degeneration in $\mathrm{db} / \mathrm{db}$ mice. Fundus examination results from FA prior to drug intervention are presented in Fig. 4A and results from after 18 weeks of treatment are presented in Fig. 4B. Compared with normal control mice (Fig. 4Aa), db/db mice exhibited deterioration of pathological neovascularization as T2DM progressed (Fig. 4Ab). However, 1.8 and $0.9 \mathrm{~g} / \mathrm{kg}$ BW TNTL treatment significantly reduced the density of vascular calibers in the fundus oculi (Fig. 4Ad and e and Bd and e).

In addition, the number of vascular calibers (i.e. pathological neovascularization) in the fundus of $\mathrm{db} / \mathrm{db}$ mice was counted to quantitatively analyze the effect of TNTL. In 
Table I. Serum lipid levels in C57BL/KsJ-db/db mice (mg/dl).

\begin{tabular}{lccccc}
\hline Group & Dose $(\mathrm{g} / \mathrm{kg})$ & $\mathrm{TC}$ & HDL & LDL & TG \\
\hline Normal & - & $51.88 \pm 11.93^{\mathrm{b}}$ & $20.25 \pm 3.21^{\mathrm{b}}$ & $11.23 \pm 4.10^{\mathrm{b}}$ & $14.73 \pm 3.39^{\mathrm{b}}$ \\
Model & - & $89.33 \pm 9.73$ & $10.01 \pm 4.33$ & $17.09 \pm 2.38$ & $25.09 \pm 4.47$ \\
Model + Met & 0.14 & $83.01 \pm 7.91$ & $17.40 \pm 1.52^{\mathrm{a}}$ & $14.88 \pm 3.25$ & $22.59 \pm 1.49$ \\
Model + TNTL & 1.8 & $69.67 \pm 5.51^{\mathrm{b}}$ & $19.86 \pm 4.02^{\mathrm{b}}$ & $12.35 \pm 1.82^{\mathrm{b}}$ & $17.17 \pm 3.40^{\mathrm{a}}$ \\
Model + TNTL & 0.9 & $75.83 \pm 9.91^{\mathrm{a}}$ & $18.67 \pm 2.33^{\mathrm{a}}$ & $14.67 \pm 3.11^{\mathrm{a}}$ & $19.92 \pm 3.01$ \\
Model + TNTL & 0.45 & $82.20 \pm 10.33$ & $17.83 \pm 3.49$ & $15.00 \pm 1.82$ & $21.97 \pm 2.01$ \\
\hline
\end{tabular}

Data are expressed as the mean \pm standard deviation. ${ }^{\mathrm{a}} \mathrm{P}<0.05,{ }^{\mathrm{b}} \mathrm{P}<0.05$ vs. model group. TC, total cholesterol; TG, triacylglycerol; $\mathrm{LDL}$, low-density lipoprotein; HDL, high-density lipoprotein; Met, metformin; TNTL, Tangningtongluo.

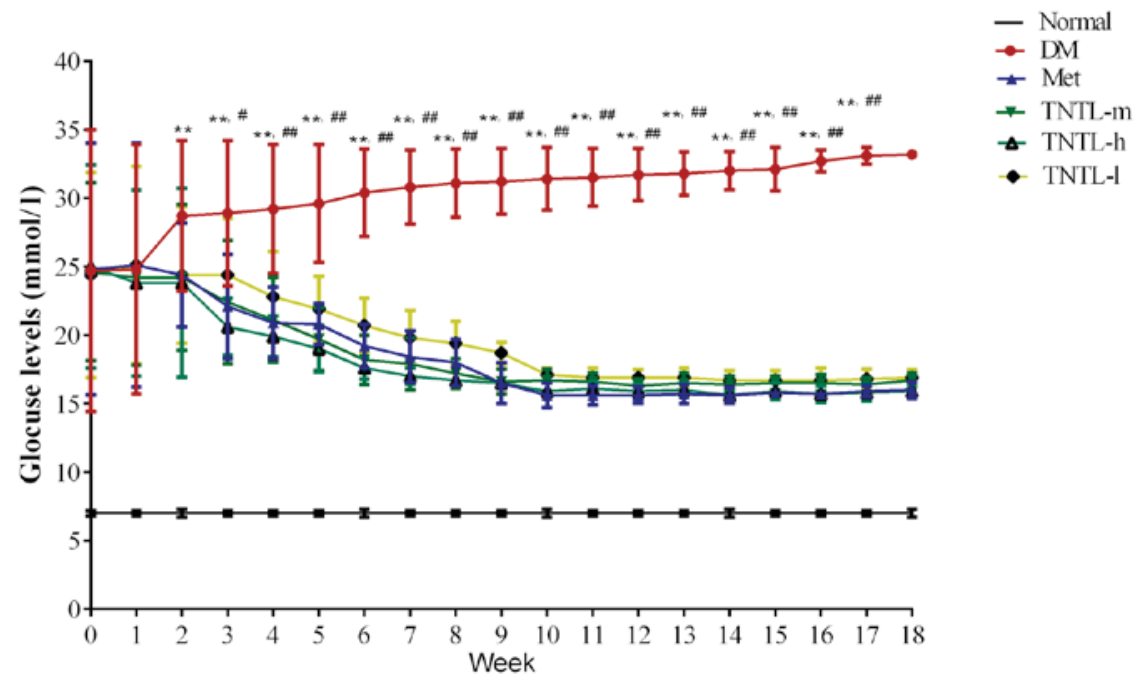

Figure 1. Fasting blood glucose levels from weeks 1 to 18. Sustained TNTL treatment exerts a time-dependent hypoglycemic effect. Data are presented as the mean \pm standard deviation. ${ }^{* *} \mathrm{P}<0.01$ vs. Normal mice, ${ }^{~} \mathrm{P}<0.05$ vs. other 4 treatment groups (Met, TNTL-m, TNTL-h and TNTL-1), ${ }^{\# \#} \mathrm{P}<0.01$ vs. other 4 treatment groups (Met, TNTL-m, TNTL-h and TNTL-1). Normal, C57BL mice; DM, untreated C57BLdb/db mice; Met, db/db mice treated with metformin; TNTL-h, TNTL-m and TNTL-1, C57BLdb/db mice treated with $1.8,0.9$ and $0.45 \mathrm{~g} / \mathrm{kg}$ body weight Tangningtongluo, respectively.

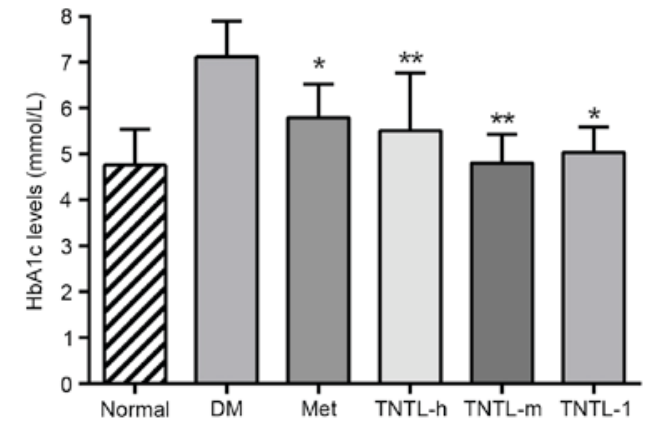

Figure 2. HbAlc in each group at week 18. Data are presented as the mean \pm standard deviation. ${ }^{*} \mathrm{P}<0.05$ vs. DM group; ${ }^{* *} \mathrm{P}<0.01$ vs. DM group. Normal, C57BL mice; DM, untreated C57BLdb/db mice; Met, C57BLdb/db mice treated with metformin; TNTL-h, TNTL-m and TNTL-1, C57BLdb/db mice treated with $1.8,0.9$ and $0.45 \mathrm{~g} / \mathrm{kg}$ body weight Tangningtongluo, respectively; HbA1c, glycated hemoglobin A1c.

diabetic mice, the number of vascular calibers increased in the model + Met group along with the progression of T2DM. Quantitative analysis of the density of vascular calibers in the

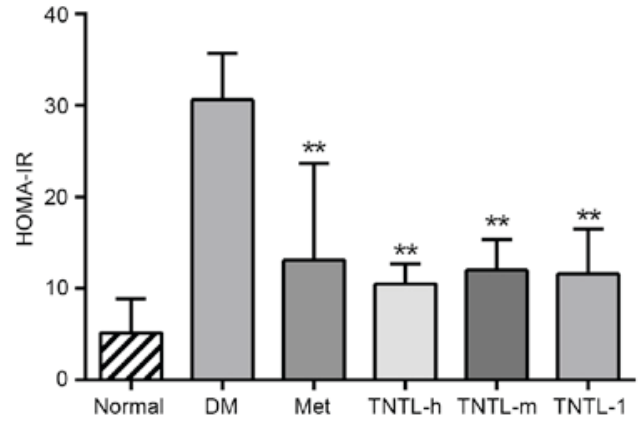

Figure 3. HOMA-IR in each group at week 18. Data are presented as the mean \pm standard deviation. ${ }^{*} \mathrm{P}<0.05$ vs. $\mathrm{DM}$ group ${ }^{* *} \mathrm{P}<0.01$ vs. $\mathrm{DM}$ group. Normal, C57BL mice; DM, untreated C57BLdb/db mice; Met, C57BLdb/db mice treated with metformin; TNTL-h, TNTL-m and TNTL-1, C57BLdb/db mice treated with $1.8,0.9$ and $0.45 \mathrm{~g} / \mathrm{kg}$ body weight Tangningtongluo, respectively; HOMA-IR, homeostatic model assessment of insulin resistance.

fundus oculi showed that TNTL significantly decreased the number of vascular calibers; in contrast, the positive control group (Met) exhibited no significant alleviation (Fig. 5). 
A a

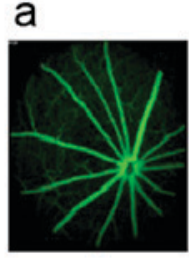

B a

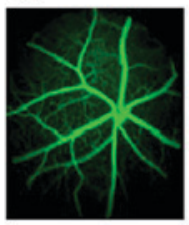

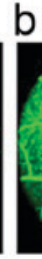

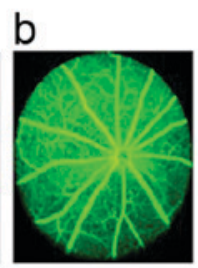

C

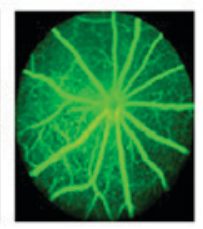

c

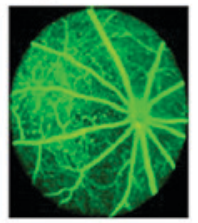

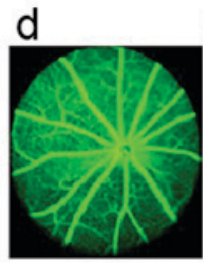
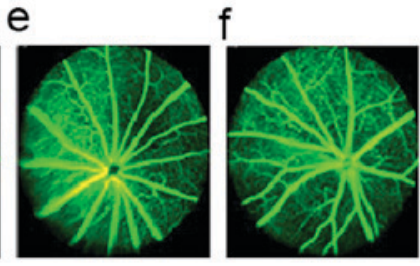

d

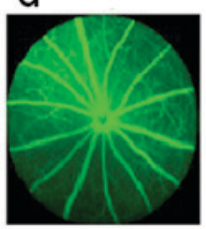

e

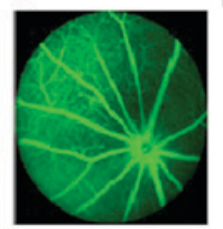

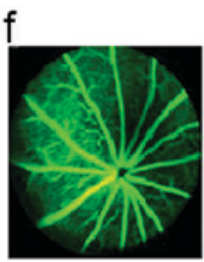

Figure 4. Retinal imaging using FA in each group. (A) Results of the fundus examination using FA prior to drug intervention in the (a) normal, (b), DM, (c), Met, (d), TNTL-h, (e) TNTL-m and (f) TNTL-1 groups. (B) Results of the fundus examination using FA after the 18-week drug intervention in the (a) normal, (b), DM, (c), Met, (d), TNTL-h, (e) TNTL-m and (f) TNTL-1 groups. Magnification, x40. Normal, C57BL mice; DM, untreated C57BLdb/db mice; Met, C57BLdb/db mice treated with metformin; TNTL-h, TNTL-m and TNTL-1, C57BLdb/db mice treated with 1.8, 0.9 and 0.45 g/kg body weight Tangningtongluo, respectively; FA, fluorescein angiography.

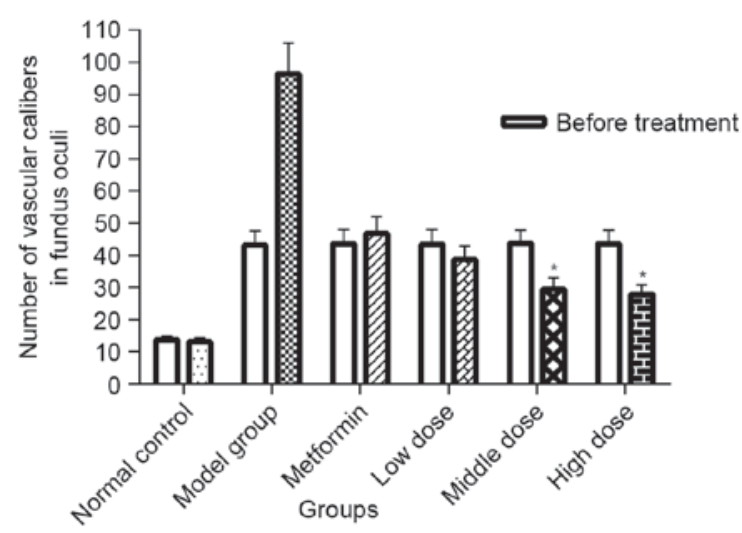

Figure 5. Quantification of retinal imaging in each group. Density of vascular calibers in the fundus oculi in each group. TNTL significantly decreased the number of small-caliber vessels. The metformin showed no significant alleviation. Data are presented as the mean \pm standard deviation. ${ }^{*} \mathrm{P}<0.05$ vs. model group. Low, middle and high dose, $0.45,0.9$ and $0.18 \mathrm{~g} / \mathrm{kg}$ body weight Tangningtongluo treatment.

Effect of TNTL on histopathological alterations in the pancreas of $\mathrm{db} / \mathrm{db}$ mice. Histopathological analyses of mouse pancreas sections are presented in Fig. 6. Compared with the normal control group, which exhibited a typical histological structure of islets in the pancreas (Fig. 6A), the diabetic control group demonstrated evidence of severe damage characterized by a reduced number and area of islets, transformation of the borders, vacuolation and degranulation of cells (Fig. 6B). Administration of 1.8, 0.9 and $0.45 \mathrm{~g} / \mathrm{kg} \mathrm{BW}$ TNTL restored the damaged islets and healed the structure (Fig. 6D-F, respectively).

Effect of TNTL on ISR-1 and p-ISR-1 protein expression. Representative western blot images of protein expression levels of ISR-1 are presented in Fig. 7A and B for liver and muscle tissue, respectively. Phosphorylation levels of ISR-1 were reduced in the $\mathrm{db} / \mathrm{db}$ mice, compared with the normal control group $(21.6 \pm 8.1 \%$ vs. $100.0 \pm 5.3 \%$ in muscle tissue, $39.8 \pm 8.9 \%$ vs. $100.0 \pm 6.4 \%$ in liver tissue). Increased p-ISR-1expression was observed in the TNTL group. The administration of TNTL led to higher expression compared with the diabetic model group, and the difference was statistically significant. As expected, the p-ISR-1 expression increased significantly in the Met group.

\section{Discussion}

TCM is used widely to treat T2DM not only in China but also worldwide $(13,20,28)$. However, the role of TCM and other herbal medicines in the management of T2DM remains to be established. For most conditions, there is a lack of scientific evidence on the underlying mechanisms of TCM methods. Over $80 \%$ of the people in developing countries depend on herbal medicine for their basic health care (19-21,29,30). Therefore, it is urgent and necessary to confirm the efficacy of TCM in the management of T2DM.

The present study aimed to evaluate the efficacy of TNTL, a TCM, to investigate the underlying mechanism. It was demonstrated that TNTL has an anti-diabetic effect in diabetic animal models, as evidenced by its hypoglycemic activity.

FA was performed to detect the progressive neural retinal pathology in $\mathrm{db} / \mathrm{db}$ mice. The speed of retinal degeneration was reduced significantly in the TNTL treatment groups, compared with the placebo and Met-treated control groups. Therefore, TNTL treatment may markedly reduce or reverse this pathological neovascularization. The TNTL-induced reduction in the risk of microvascular complications (diabetic retinopathy) demonstrated the clinical advantage of comprehensive treatment effects of TCM. Furthermore, the present study indicated that TNTL treatment reduced insulin resistance, as evidenced by the decrease in HOMA-IR. This finding may partially explain the mechanism of the hypoglycemic effect. Collectively, these results demonstrated that TNTL exerts a strong hypoglycemic effect and reverses retinal degeneration. Therefore, TNTL may be beneficial in preventing the progression of T2DM. 

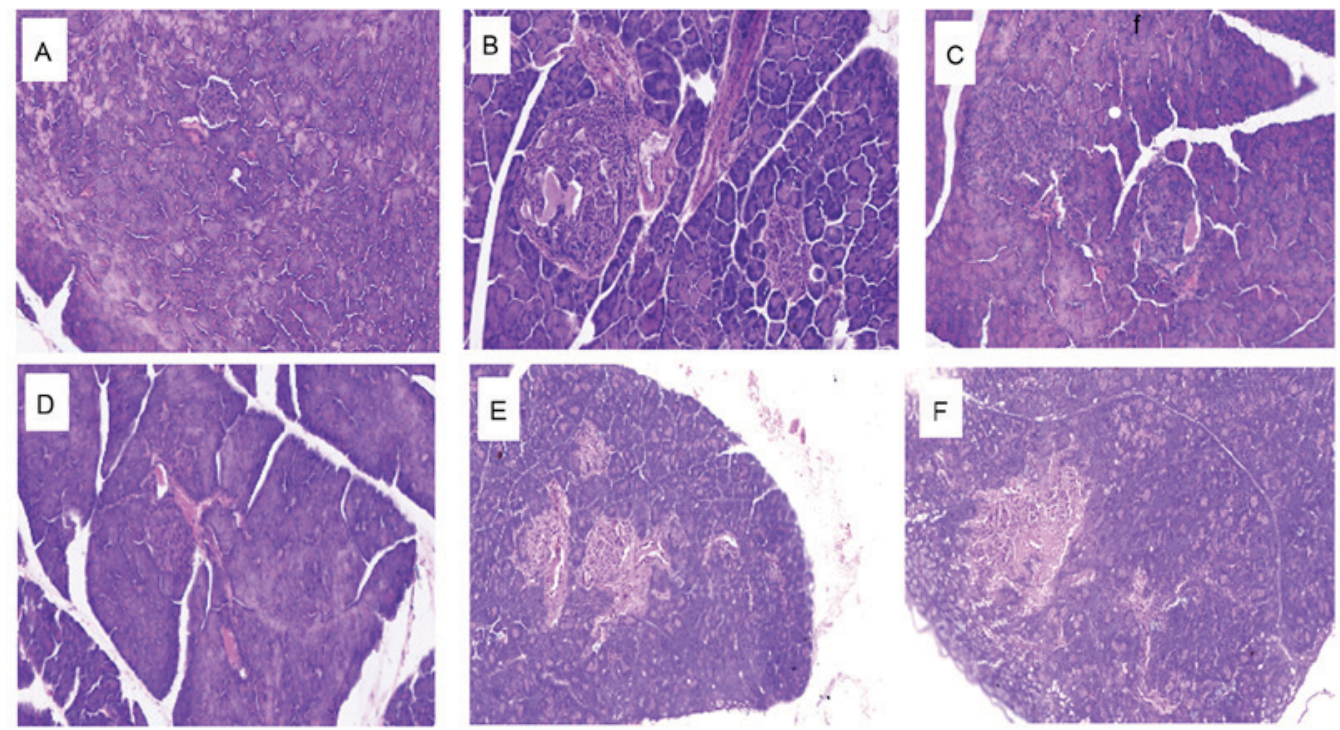

Figure 6. Histopathological alterations in the pancreas in each group. Representative images of mice in the (A) normal control, (B) diabetic control, (C) metformin and (D) 1.8 , (E) 0.9 and (F) $0.45 \mathrm{~g} / \mathrm{kg}$ body weight Tangningtongluo-treated groups.

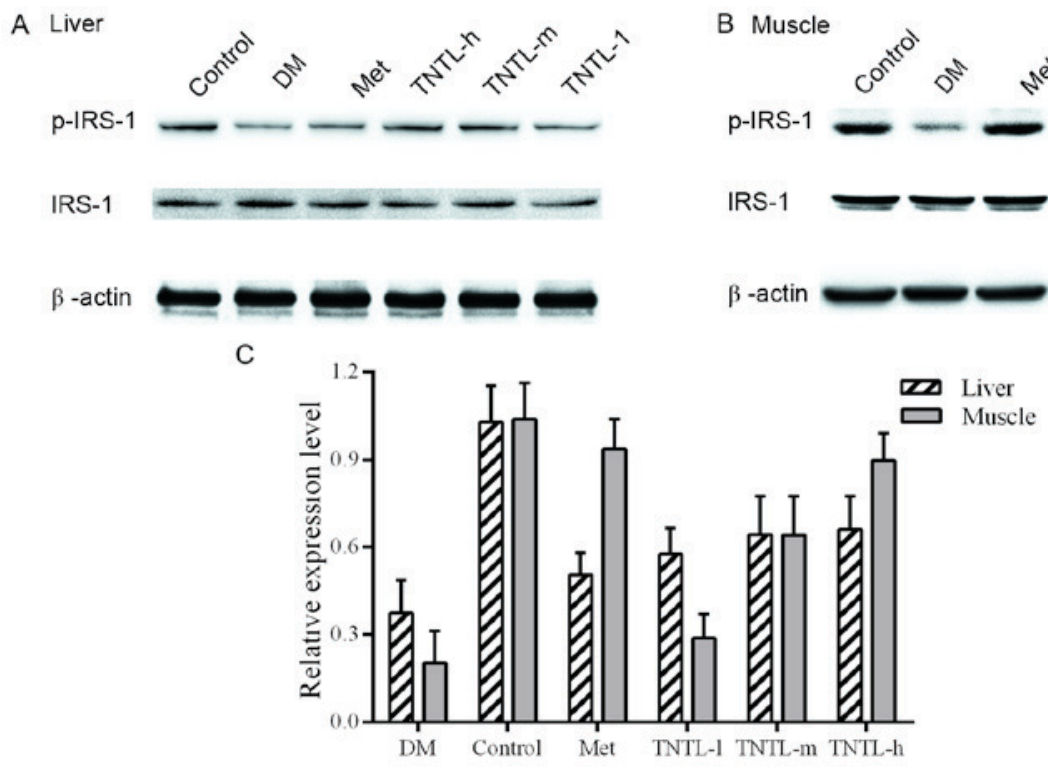

Figure 7. Protein expression levels of IRS-1 and p-IRS-1 in each group. Representative western blot images of (A) liver and (B) muscle tissue, and (C) quantification of IRS-1 and p-IRS-1 protein expression levels in each group. $\beta$-actin served as an internal control. Met, metformin; IRS, insulin receptor substrate-1; p, phosphorylated; DM, untreated C57BLdb/db mice; TNTL-h, TNTL-m and TNTL-1, C57BLdb/db mice treated with 1.8, 0.9 and 0.45 g/kg body weight Tangningtongluo.

ISR-1, a docking protein that engages downstreammolecules in the insulin signaling network, is a key mediator of insulin resistance (31-33). IRS-1 is downregulated in the liver and skeletal muscle of diabetic animals (34). The protein expression levels of ISR-1 and p-ISR (its active form) were investigated to elucidate the molecular mechanism underlying the alleviation of insulin resistance and the hyperglycemic effect. The present study confirmed that TNTL administration elevated p-IRS-1 protein expression levels in the liver and skeletal muscle of $\mathrm{db} / \mathrm{db}$ mice compared with non-diabetic mice, suggesting that TNTL may restore the impaired insulin signaling pathway. The result indicated that the phosphorylation of IRS-1 caused by TNTL administration was strongly associated with the improvement in insulin resistance.

In conclusion, the results of the present study demonstrated that TNTL exerts a strong hypoglycemic effect, reduces insulin resistance and reverses retinal degeneration. In addition, it was demonstrated that the anti-diabetic effects of TNTL are strongly involved in the regulation of ISR-1 phosphorylation, suggesting a potential mechanism. Therefore, TNTL may be an anti-diabetic agent for the effective treatment of T2DM and an alternative therapeutic to current drugs. Further investigations are required to elucidate the precise molecular mechanism by which TNTL exerts anti-diabetic effects and reverses retinal degeneration. 


\section{Acknowledgements}

The present study was supported by the Beijing Science and Technology Projects (grant no. Z141100002114044), the National Major Drug Discovery Projects (grant no. 2012ZX09501001 and 2012ZX09301002); The Open Project of Key Laboratory of Zunyi Medical University (ZY-2015-01); and Guizhou Bailing Pharmaceutical Co., Ltd. The authors thank scientific editors at American Journal Experts for providing professional English language editing of this paper.

\section{References}

1. Chan JC, Malik V, Jia W, Kadowaki T, Yajnik CS, Yoon KH and Hu FB: Diabetes in Asia: Epidemiology, risk factors, and pathophysiology. Jama 301: 2129-2140, 2009.

2. Chan JC, Zhang Y and Ning G: Diabetes in China: A societal solution for a personal challenge. Lancet Diabetes Endocrinol 2: 969-979, 2014.

3. Ning G and Bloomgarden Z: Diabetes in China: Prevalence, diagnosis, and control. J Diabetes 5: 372, 2013.

4. Weng J and Bi Y: Diabetes in China: The challenge now. J Diabetes Investig 1: 170-171, 2010.

5. L'Heveder R and Nolan T: International Diabetes Federation. Diabetes Res Clin Pract 101: 349-351, 2013.

6. Yang W, Lu J, Weng J, Jia W, Ji L, Xiao J, Shan Z, Liu J, Tian H, $\mathrm{Ji} Q$, et al: Prevalence of diabetes among men and women in China. N Engl J Med 362: 1090-1101, 2010.

7. Yu S, Sun Z, Zheng L, Guo X, Yang H and Sun Y: Prevalence of Diabetes and Impaired Fasting Glucose in Hypertensive Adults in Rural China: Far from Leveling-Off. Int J Environ Res Public Health 12: 14764-14779, 2015.

8. Hu D, Sun L, Fu P, Xie J, Lu J, Zhou J, Yu D, Whelton PK, He J and Gu D: Prevalence and risk factors for type 2 diabetes mellitus in the Chinese adult population: The InterASIA Study. Diabetes Res Clin Pract 84: 288-295, 2009.

9. Hu D, Fu P, Xie J, Chen CS, Yu D, Whelton PK, He J and $\mathrm{Gu}$ D; MS for the InterASIA Collaborative Group: Increasing prevalence and low awareness, treatment and control of diabetes mellitus among Chinese adults: The InterASIA study. Diabetes Res Clin Pract 81: 250-257, 2008.

10. Jia W: Diabetes: A challenge for China in the 21st century. Lancet Diabetes Endocrinol 2: e6-e7, 2014.

11. Chi C, Lee JL, Lai JS, Chen SC, Chen CY and Chang SK: Utilization of Chinese medicine in Taiwan. Altern Ther Health Med 3: 40-53, 1997.

12. Hesketh T and Zhu WX: Health in China. Traditional Chinese medicine: One country, two systems. BMJ 315: 115-117, 1997.

13. Pang B, Zhou Q, Zhao TY, He LS, Guo J, Chen HD, Zhao LH and Tong XL: Innovative thoughts on treating diabetes from the perspective of traditional Chinese medicine. Evid Based Complement Alternat Med 2015: 905432, 2015.

14. Tang C, Ye Y, Feng Y and Quinn RJ: TCM, brain function and drug space. Nat Prod Rep 33: 6-25, 2016.

15. Tong XL, Dong L, Chen L and Zhen Z: Treatment of diabetes using traditional Chinese medicine: Past, present and future. Am J Chin Med 40: 877-886, 2012.

16. Wang P, Xu Q, Sun Q, Fan FF, Guo XR and Guo F: Assessment of the reporting quality of randomized controlled trials on the treatment of diabetes mellitus with traditional chinese medicine: A systematic review. PLoS One 8: e70586, 2013.

17. Zhang TT and Jiang JG: Active ingredients of traditional Chinese medicine in the treatment of diabetes and diabetic complications. Expert Opin Investig Drugs 21: 1625-1642, 2012.

18. Jiang HL, Niu JJ, Zhang WF, Huang WJ, Zhou MY, Sha WJ, Li JY, Li FF, Zhu T, Xia X, et al: The role of central nervous system on hypoglycemia and the feasibility of the brain theory in traditional Chinese medicine on treatment of diabetes mellitus. J Integr Med 12: 1-6, 2014.
19. Lao L and Ning Z: Integrating traditional Chinese medicine into mainstream healthcare system in Hong Kong, China-A model of integrative medicine in the HKU-SZ Hospital. J Integr Med 13: 353-355, 2015.

20. Li Y, Wu T, Shang H and Yang K; all subcentres attending the Chinese EBM Working Meeting in December 2008: Strategies for promoting the development of evidence-based medicine in China. J Evid Based Med 2: 47-52, 2009.

21. Liao PY, Hsu PC, Chen JM, Chiang JY and Lo LC: Diabetes with pyogenic liver abscess-A perspective on tongue assessment in traditional Chinese medicine. Complement Ther Med 22: 341-348, 2014.

22. Lin YJ, Ho TJ, Yeh YC, Cheng CF, Shiao YT, Wang CB, Chien WK, Chen JH, Liu X, Tsang H, et al: Chinese herbal medicine treatment improves the overall survival rate of individuals with hypertension among type 2 diabetes patients and modulates in vitro smooth muscle cell contractility. PLoS One 10: e0145109, 2015.

23. Wu Q and Fan $\mathrm{H}$ : The research for the clinical curative effect through combing traditional Chinese medicine with insulin to cure diabetes. Pak J Pharm Sci 27 (4 Suppl): S1057-S1061, 2014.

24. Cheng L, Meng XB, Lu S, Wang TT, Liu Y, Sun GB and Sun XB: Evaluation of hypoglycemic efficacy of tangningtongluo formula, a traditional Chinese Miao medicine, in two rodent animal models. J Diabetes Res 2014: 745419, 2014.

25. Chen H, Charlat O, Tartaglia LA, Woolf EA, Weng X, Ellis SJ, Lakey ND, Culpepper J, Moore KJ, Breitbart RE, et al: Evidence that the diabetes gene encodes the leptin receptor: Identification of a mutation in the leptin receptor gene in $\mathrm{db} / \mathrm{db}$ mice. Cell 84: 491-495, 1996.

26. Kodama H, Fujita M and Yamaguchi I: Development of hyperglycaemia and insulin resistance in conscious genetically diabetic (C57BL/KsJ-db/db) mice. Diabetologia 37: 739-744, 1994.

27. Wallace TM, Levy JC and Matthews DR: Use and abuse of HOMA modeling. Diabetes Care 27: 1487-1495, 2004.

28. Poon TY, Ong KL and Cheung BM: Review of the effects of the traditional Chinese medicine Rehmannia Six Formula on diabetes mellitus and its complications. J Diabetes 3: 184-200, 2011.

29. Ji L, Tong X, Wang H, Tian H, Zhou H, Zhang L, Li Q, Wang Y, Li H, Liu M, et al: Efficacy and safety of traditional chinese medicine for diabetes: A double-blind, randomised, controlled trial. PLoS One 8: e56703, 2013.

30. Wei J, Wu R and Zhao D: Analysis on traditional Chinese medicine syndrome elements and relevant factors for senile diabetes. J Tradit Chin Med 33: 473-478, 2013.

31. Angiolillo DJ, Bernardo E, Zanoni M, Vivas D, Capranzano P, Malerba G, Capodanno D, Prandini P, Pasquali A, Trabetti E, et al: Impact of insulin receptor substrate-1 genotypes on platelet reactivity and cardiovascular outcomes in patients with type 2 diabetes mellitus and coronary artery disease. J Am Coll Cardiol 58: 30-39, 2011.

32. Orkunoglu Suer FE, Mergen H, Bolu E and Ozata M: Molecular scanning for mutations in the insulin receptor substrate-1 (IRS-1) gene in Turkish with type 2 diabetes mellitus. Endocr J 52: 593-598, 2005.

33. Zhang Y, Sun CM, Hu XQ and Zhao Y: Relationship between melatonin receptor $1 \mathrm{~B}$ and insulin receptor substrate 1 polymorphisms with gestational diabetes mellitus: A systematic review and meta-analysis. Sci Rep 4: 6113, 2014.

34. Karolina DS, Armugam A, Tavintharan S, Wong MT, Lim SC, Sum CF and Jeyaseelan K: MicroRNA 144 impairs insulin signaling by inhibiting the expression of insulin receptor substrate 1 in type 2 diabetes mellitus. PLoS One 6: e22839, 2011. 